\title{
ELECTROPHYSIOLOGICAL IMAGING OF VOLUMETRIC INFARCT BORDER USING A SPATIO-TEMPORAL $L P$-NORM CONSTRAINT
}

\author{
Azar Rahimi, Jingjia Xu, Linwei Wang \\ Galisano College of Computing and Information Sciences \\ Computational Biomedicine Labratory \\ Rochester Institute of Technology
}

\begin{abstract}
Imaging the bioelectrical source distribution along the infarct border is of important therapeutic potential as infarct border is the common site for reentry circuits leading to life-threatening arrhythmias. Although it can be tackled by computational reconstruction of volumetric cardiac source using body-surface voltage data, the problem is notoriously ill-posed without a unique solution. Progress towards 3D cardiac source reconstruction is further hindered by the complex spatio-temporal property of cardiac current sources, which decides that common $L 1$ and $L 2$-norm constraints are not proper because the underlying assumption is either too focal or too smooth to reflect the property of the source distribution. In this work, we propose a general regularization with $L p$-norm $(1<p<2)$ constraint to bridge the gap between $L 1$ and $L 2$ solutions in cardiac source reconstruction along the infarct border. We further incorporate temporal information into $L p$-norm constraint to promote consistency over time as well as robustness to noise. In a set of computer-simulated and real-data experiments, we demonstrate the superiority of the proposed method in reflecting the complex spatial structure of source distribution along 3D infarct border during the ST-segment of an ECG cycle, over $L 1$ or $L 2$ counterparts.
\end{abstract}

Index Terms - Forward and inverse bioelectrical problem, volumetric cardiac current source imaging, $L p$-norm, singular value decomposition

\section{INTRODUCTION}

Heterogeneous infarct border, created during ischemic cardiomyopathy with a mix of necrotic and viable tissues, is the common site for re-entrant circuits that can initiate and maintain life-threatening ventricular arrhythmia [1]. Characterization of both the anatomy and elecrophysiology of this heterogeneous region is of great importance for arrhythmia treatment [1]. Although the anatomy of infarct border can be characterized using structural imaging such as delayed enhanced MRI, evaluating its electrophysiological aspects is still restricted to an invasive catheter mapping that is surfacebased with limited spatial resolution. This gap in practice raises a great demand for a noninvasive and transmural imaging modality that can reveal volumetric bioelectrical source activities that are bordering the transmural infarct.

Computational electrphysiological imaging tackles this problem by mathematically reconstructing the 3D timevarying bioelectrical currents using noninvasive electrocardiographic (ECG) data. The biophysical model between the current sources and the body-surface voltages follows the quasi-static electromagnetsm on anatomical regions of heart and torso [2]. With proper numerical methods and discretization of the current source $\mathbf{v}$ and measurement fields $\mathbf{b}$, this biophysical model renders a linear relationship: $\mathbf{b}=\mathbf{H v}$, where $\mathbf{H}$ is specific to subject's anatomy and typically assumed time-invariant. However, this inverse problem suffers from both mathematical and physical ill-posedness [3]. The mathematical ill-posedness is caused by the limited number of field measurements compared to the large number of current sources. The physical ill-posedness is unique to the underlying biophysics of this problem: in a quasi-static electromagnetic field, different configurations of 3D sources may produce the same surface measurements [2]. Therefore, if the solution is sought transmurally, this inverse problem is intrinsically ill-posed without a unique solution in its unconstrained form. Proper assumptions on the solutions must be made in order to guarantee a unique solution.

It is notable that the same biophysics governs also the relationship between current sources in the brain and surface voltage data measured on the skull. However, progress in the two fields have seen substantial differences. Numerous approaches (based on $L 2$ and $L 1$-norm) are developed in estimating the volumetrically distributed current sources of the brain $[4,5,6]$ while in the heart the reconstructions are mainly restricted to the heart surfaces $[7,8]$. We hypothesize that the difference in the challenge of these two problems is partly caused by the different spatio-temporal properties of the cardiac and neural current sources. Neural current sources are often compact, and the research interest is in locating the peak active region in the brain at any given condition. In comparison, cardiac current sources undergo a complex spatiotemporal process in each cardiac cycle, starting from a few fo- 


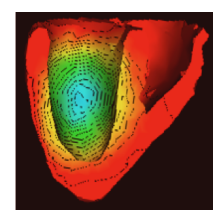

(A)
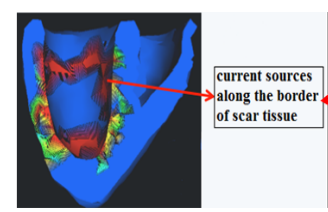

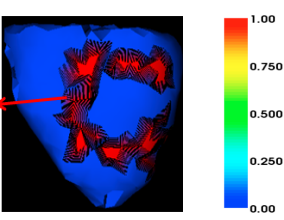

(B)
Fig. 1. Illustration of (A) action potential and (B) the spatial structure of current sources distributed along the infarct border during ST-segment of an ECG cycle.

cal sites and then propagating throughout the myocardium. In particular, in an infarcted heart, active sources will be concentrated along the infarct border after depolarization and before the repolarization (ST-segment in an ECG cycle), as shown in Fig 1B, while a healthy heart is expected to go through a stage without current flow. This unique spatial property of cardiac current sources decides that $L 2$ or $L 1$ regularization will produce a solution that is either too smeared or too focal to reveal the underlying source activity. This is shown in a few transmural cardiac source imaging methods that essentially amount to quadartic approaches using either simple $L 2$ priors [9] or complex physiological priors derived from propagation models [10], where the solutions are often diffusive due to the inherent heuristic assumption of smoothness.

Based on this observation, we propose a simple yet novel application of general regularization based on $L p$ norm $(1<p<2)$ to $3 \mathrm{D}$ cardiac source imaging. To promote the temporal consistency and stability of the reconstruction during the ST-segment at the presence of measurement noise, we assume that current sources share the same temporal basis functions as the surface measurements. Decomposing the surface measurement into spatial and temporal components using singular value decomposition (SVD), we apply $L p$-norm regularization to the remaining spatial component. In a set of phantom and real-data experiments, our method is demonstrated to outperform its $L 1$ and $L 2$ counterparts in imaging the complex structure of the sources distributed along the infarct border during ST-segment.

\section{METHODOLOGY}

The quasi-static electromagnetism [2] explains the relation between the potential distribution within the torso volume and the cardiac current sources as:

$$
\begin{gathered}
\sigma_{b l k} \nabla^{2} \phi_{e}(\mathbf{r})=\nabla \cdot\left(-\mathbf{D}_{i n t} v(\mathbf{r})\right), \forall \mathbf{r} \in \Omega_{h} \\
\sigma \nabla^{2} \phi(\mathbf{r})=0, \forall \mathbf{r} \in \Omega_{t / h}
\end{gathered}
$$

where the Poisson equation (1) describes, on a bidomain heart model, how the extracellular potential $\phi_{e}$ within the heart volume $\Omega_{h}$ originates from the current sources $\mathbf{D}_{\text {int }} v$ where $v$ is the targeted solution that represents the gradient of the action potential and $\mathbf{D}_{\text {int }}$ is the anisotropic intracellular conductivity tensor. $\sigma_{b l k}$ is the isotropic bulk conductivity. The Laplace equation (2) describes, on the monodomain torso model, how the potential $\phi$ distributes within the torso volume $\Omega_{t / h}$ external to the heart with conductivity $\sigma$. It has been previously shown that, using proper numerical methods such as mesh free and boundary element methods, one can numerically solve equation $(1,2)$ on a subject heart-torso model, and obtain linear relationship: $\mathbf{b}=\mathbf{H v}$ [11].

$L p$-SVD Regularization. As mentioned earlier, reconstructing 3D current sources from ECG data is a highly ill-posed inverse problem with non-unique solution in its most unconstrained form. Furthermore, complex spatial distribution of cardiac current sources conflicts with a focal $L 1$ or smooth $L 2$ constraints. To estimate the complex spatial structure of current sources, we first assume that current sources share the same temporal basis functions as ECG measurements. These temporal basis functions can be obtained by different transformations, and in this study we consider taking the singular value decomposition of ECG measurements: $\mathbf{b}=\mathbf{U S T}^{\prime}$, where $\mathbf{T}=\left[\mathbf{t}_{\mathbf{1}}, \mathbf{t}_{\mathbf{2}}, \ldots, \mathbf{t}_{\mathbf{k}}\right]$ defines the orthonormal temporal basis functions. Projecting both ECG measurements and current sources onto the basis functions $\mathbf{T}$, the new variables $\tilde{\mathbf{b}}=\mathbf{b T}$ and $\tilde{\mathbf{v}}=\mathbf{v T}$ are obtained that represent the corresponding projection coefficients. We then apply $L p$-norm regularization to reconstruct new variable $\tilde{\mathbf{v}}$ from $\tilde{\mathbf{b}}$. In this way, the $L p$ regularization problem can be formulated as

$$
\min _{\tilde{\mathbf{v}}}\|\tilde{\mathbf{b}}-\mathbf{H} \tilde{\mathbf{v}}\|_{2}^{2}+\lambda\|\tilde{\mathbf{v}}\|_{p}, \quad\|\tilde{\mathbf{v}}\|_{p}=\left(\sum_{i=1}^{n}\left[\tilde{v}_{i}\right]^{p}\right)^{1 / p} \text { (3) }
$$
whêre $n$ is the dimension of $\tilde{\mathbf{v}}$, i.e., the number of discrete mesh-free nodes used to represent the ventricular myocardium and $1<p<2$. The regularization parameter $\lambda$ is selected impirically for different $p$ values. Solving the regularization problem (3) using second order cone programming, the optimal coefficient $\tilde{\mathbf{v}}$ would be obtained. The current sources $\mathbf{v}$ are then calculated as linear combinations of the temporal basis functions: $\mathbf{v}=\tilde{\mathbf{v}} \mathbf{T}$.

By solving the regularization (3) in the transformed domain spanned by the temporal basis of the measurement $\mathbf{b}$, we promote the temporal consistency of the reconstructed sources at the presence of measurement noises so the reconstructed sources would not jump over neighboring regions at consecutive time instants. Additionally, solving the problem in singular vector domain (3) have an advantage of reducing the size of the problem and increasing the robustness to noise. In typical situations where the number of time instants for $\mathbf{b}$ is in the order of hundreds, the leading singular vectors of $\mathbf{b}$ are less than 10 (accounting for $\geq 90 \%$ of the data variation). Thus, solving (3) only for the first 10 leading singular vectors results in $\geq 95 \%$ reduction of the problem size as well as an increased robustness to noise.

\section{RESULTS}

\subsection{Computer-Simulated Electrical Activity}

We consider synthetic experiments on an image-derived human heart-torso model. The torso surface is represented by 


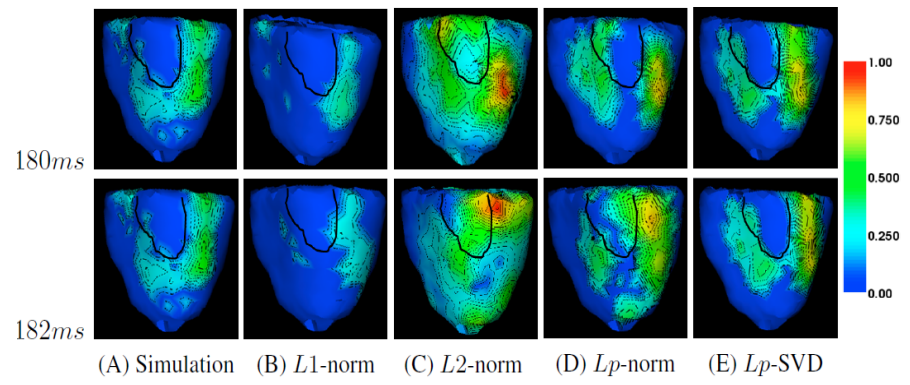

Fig. 2. Estimation of current sources localized along the infarct border using $L p$-SVD regularization vs. $L p(p=1.5)$, $L 1$ and $L 2$-norm counterparts at two consecutive time samples during ST-segment of an ECG cycle.

triangulated elements with 370 vertices. The ventricular myocardium is represented by 1019 evenly distributed nodes. Realistic transmural action potential propagation is simulated by the Aliev-Panfilov [12] model. The corresponding ECG measurements on the body-surface are simulated and the STsegment time frames are selected and corrupted with $20 \mathrm{~dB}$ noise as inputs to reconstruct the current source distribution. The accuracy of 3D source estimation is evaluated using the source overlap $(\mathrm{SO})$ defined as the ratio of the intersection to the union between the estimated and the true current sources averaged over a cardiac cycle. We also perform comparison studies of the proposed $L p$-SVD method with $L 1$ and $L 2$ norm methods in 3D source imaging.

Impact of Time Incorporation on Lp-norm Regularization. Fig 2 shows an example of current source distribution along the infarct border that extends from basal to mid anterior and anterolateral LV (black contour) at 2 consecutive time samples during ST-segment. $L 1$ regularization produces a scattered solution with $\mathrm{SO}=0.08$ and 0.06 , respectively. $L 2$ regularization produces a diffused solution with $\mathrm{SO}=0.26$ and 0.24 . Neither methods are able to capture the structure of the current sources along the infarct border. In comparison, the $L p$ regularization $(p=1.5)$ obtains $\mathrm{SO}=0.35$ and $=0.31$ that are closer to the ground truth but are inconsistent between the 2 time instants. $L p$-SVD $(p=1.5)$ provides $\mathrm{SO}=0.39$ and 0.38 in regions consistent with the true infarct border. The advantage of a $L p$ prior over $L 1$ or $L 2$ is evident in reflecting the important complex spatial shape of source distribution, and the incorporation of temporal constraints (the proposed SVD) is shown to further improve the temporal consistency of the solution especially at the presence of measurement noises.

Impact of $p$ Value on Imaging Infarct Regions with Different Sizes. We consider 72 experiments with infarcts located at different areas and sized from $1 \%$ to $45 \%$ of LV. Fig 3 presents SO mean and standard deviation between the true and estimated infarct border during ST segment obtained using $L p$-SVD, as $p$ increases from 1 to 2 and as the size of infarct border increases. The sparse solution produced by $L 1$-norm regularization, though produces low false-positives, also has a high under-estimation. On the other extreme, though the smeared

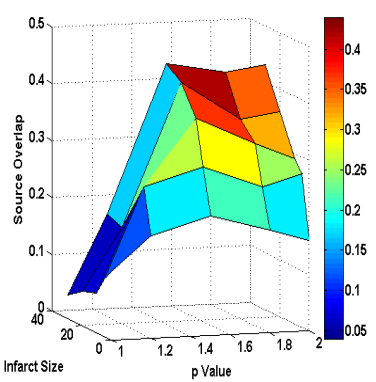

Fig. 3. Comparison of segment overlap (SO) with respect to infarct border size.

solution of $L 2$-norm regularization is able to detect the majority of the true active sources, it tends to have a high overestimation due to the diffused solution. Therefore, for source region of all sizes, we can observe an increase followed by a decrease in the SO value when $p$ increases from 1 to 2 , with the maximum SO obtained at $p=1.5-1.6$.

For all the 72 cases, the mean and standard deviation of SO for our method, $L 1$ and $L 2$ are $0.37 \pm 0.04,0.07 \pm 0.05$ and $0.32 \pm 0.09$, respectively. Paired student's t-test shows that the accuracy of the proposed method in preserving the structure of the infarct border is significantly higher than that of $L 1(p<0.0001)$ and $L 2(p<0.005)$.

\subsection{Real-data Experiments on Post-infarction Patients}

Because of the important therapeutic value of the infarct border and the promising results obtained from our initial synthetic experiments, we continue to conduct an initial real-data experiment on a post-infarction human subject to assess the feasibility of the proposed $L p$-norm method in detecting current sources along the infarct border during the ST-segment of an ECG cycle.

Experimental data were collected from a patient with prior myocardial infarction [13]. MRI scan of the patient has 8$\mathrm{mm}$ inter-slice spacing and $1.33 \mathrm{~mm} /$ pixel in-plane resolution. Body-surface ECG maps were recorded by Dalhousie University standards [14] at 123 known anatomical sites and interpolated to 370 nodes of the Dalhousie torso model; each ECG recording consists of a single averaged PQRST complex sampled at $2 \mathrm{k} \mathrm{Hz}$. Gold standards of the infarct were provided by cardiologists who examined the late Gadolinium enhanced MR scans of the patient, and were provided in terms of the location and size of the infarct with regard to the 17-segment division of the LV according to AHA standards [15]. Specifically, according to the gold standard, the infarct center is located at segment 10 and 11 of the LV (highlighted with black contour on Fig 4) and extends to the neighboring segments.

$L 1$-norm (Fig 4A) results in a very sparse solution scattered far from the infarct center during the ST-segment. $L 2$ norm estimates (Fig 4B) are diffused and cover the infarct center. Neither methods are able to reveal any information on the location or the structure of the infarct border. The 


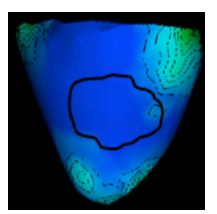

(A) $L 1$-norm

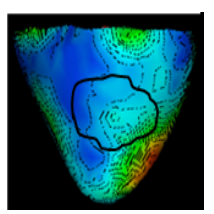

(B) $L 2$-norm

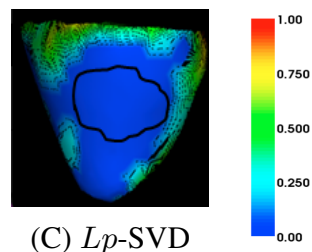

(C) $L p$-SVD
[5] I. F. Gorodnitsky and B. D. Rao, "Sparse signal reconstruction from limited data using FOCUSS: a reweighted minimum norm algorithm," IEEE Transactions on Signal Processing, vol. 45, pp. 600-616, 1997.

[6] K. Uutela, M. S. Hamalainen, and E. Somersalo, "Visualization of magnetoencephalographic data using minimum current estimates," NeuroImage, pp. 173-180, 1999.

[7] Y. Rudy and B. Messinger-Rapport, "The inverse problem of electrocardiography: solutions in terms of epicardial potentials," Critical Reviews in Biomedical Engineering, vol. 16, pp. 215-268, 1988.

[8] G. Huiskamp and F. Greensite, "A new method for myocardial activation imaging," IEEE Transactions on Biomedical Engineering, vol. 44, no. 6, pp. 446, 1997.

The inverse problem of volumetric cardiac source imaging is notoriously ill-posed without a unique solution. Progress towards volumetric cardiac source reconstruction is further hindered by the complex spatio-temporal property of cardiac current sources, which decides that common $L 1$ and $L 2$-norm constraints are no longer proper because the underlying assumption is either too focal or too smooth to reflect the property of the source distribution. We proposed a novel yet simple application of $L p$-norm regularization to bridge the gap between $L 1$ and $L 2$-norm, and show its potential in imaging cardiac current source distributions along the infarct border that are of important therapeutic information. We further incorporated temporal information using SVD to improve temporal stability and robustness to noise.

Our experiment results show that, for sources with different sizes, the highest accuracy is obtained at an empirically optimal value $p=1.5-1.6$ (Fig 3 ). This set of study proves the feasibility and paves the road for our future work to cast the problem into a Bayesian framework and treat $p$ as a hyperparameter to be estimated simoultanously from the data.

\section{REFERENCES}

[1] J. M. de Bakker, F. J. van Capelle, M. J. Janse, S. Tasseron, J. T. Vermeulen, N. de Jonge, and J. R. Lahpor, "Slow conduction in the infarcted human heart. zigzag course of activation," Circulation, vol. 88, no. 3, pp. 915-926, 1993.

[2] R. Plonsey, Bioelectric phenonmena, McGraw Hill, New York, 1969.

[3] I. F. Gorodnitsky, "Can compact generators of eeg/meg fields be identified uniquely?," Advances in Biomagnetism Research, 1998.

[4] J. Z. Wang, S. J. Williamson, and L. Kaufman, "Magnetic source imaging based on the minimum-norm leastsquares inverse," Brain Topography, vol. 5, pp. 365371, 1993.
9] Z. Liu, C. Liu, and B. He, "Noninvasive reconstruction of three-dimensional ventricular activation sequence from the inverse solution of distributed equivalent current density," IEEE Transactions on Medical Imaging, vol. 25, no. 10, pp. 1307-1318, 2006.

[10] L. Wang, H. Zhang, K. Wong, H. Liu, and P. Shi, "Physiological-model-constrained noninvasive reconstruction of volumetric myocardial transmembrane potentials," IEEE Transactions on Biomedical Engineering, vol. 57, no. 2, pp. 296-315, 2010.

[11] L. Wang, H. Zhang, K. Wong, H. Liu, and P. Shi, "Electrocardiographic simulation on personalized heart-torso structures using coupled meshfree-bem platform.," International Journal of Functional Informatics and Personalized Medicine, vol. 57, pp. 296-315, 2009.

[12] Rubin Aliev and Er V. Panfilov, "A simple two-variable model of cardiac excitation," 1996.

[13] "Physionet/computers in cardiology challenge 2007: Electrocardiographic imaging of myocardial infarction.," 2007.

[14] L. M. Title, S. E. Iles, M. J. Gardner, C. J. Penney, J. C. Clements, and B. M. Horacek, "Quantitative assessment of myocardial ischemia by electrocardiographic and scintigraphic imaging," Journal of Electrocardiology, vol. 36(Suppl), pp. 17-26, 2003.

[15] M. D. Cerqueira, N. J. Weissman, V. Dilsizian, A. K. Jacobs, S. Kaul, W. K. Laskey, D. J. Pennell, J. A. Rumberger, T. Ryan, and M. S. Verani, "Standardized myocardial segmentation and nomenclature for tomographic imaging of the heart," Circulation, vol. 105, pp. 539-542, 2002. 